\title{
Comparison of Local versus General Anaesthesia for Carpal Tunnel Release
}

\author{
Karaca S ${ }^{1 *}$, Akpolat AO', Oztermeli A', Bildik \\ $\mathrm{C}^{1}$, Erdem $\mathrm{MN}^{2}$ and Saygi $\mathrm{B}^{1}$ \\ ${ }^{1}$ Department of Orthopaedics and Traumatology, Fatih \\ Sultan Mehmet Training and Research Hospital, Turkey \\ ${ }^{2}$ Department of Orthopaedics and Traumatology, Hisar \\ Intercontinental Hospital, İstanbul, Turkey \\ *Correspondling author: Sinan Karaca, Fatih Sultan \\ Mehmet Training and Research Hospital, Orthopaedics \\ and Traumatology Clinic, 34856 Ataşehir/İstanbul, \\ Turkey
}

Received: December 11, 2016; Accepted: March 09, 2017; Published: March 15, 2017

\begin{abstract}
Purpose: Our aim was to compare the clinical results of patients had carpal tunnel release surgery under local anesthesia and general anesthesia.

Methods: We studied visual analogue scores and visual analogue patient satisfaction scores of 148 patients who underwent carpal tunnel release surgery. 100 patients who met the inclusion criteria were included in this study. There were 12 males 38 females in general anesthesia group and 15 males 35 females in local anesthesia group. The mean age in general anesthesia group was 51.4 (31-78) and in local anesthesia group was 54.4 (28-81).
\end{abstract}

Results: Visual analogue scores in general anesthesia group was 7.9 and decreased 2.9 postoperatively in local anesthesia group visual analogue score was 8.0 and decreased 2.9 postoperatively $(p>0.05)$. Visual analogue patient satisfaction score was 7.6 in general anesthesia group and 7.9 in local anesthesia group $(p>0.05)$.

Conclusion: A high percentage of patients reported high levels of satisfaction, excellent results and improvements in their quality of life in both group. As the VAS and VAPSS were considered the primary outcome measure, the results of our study show that the type of anaesthesia has no effect on the results of surgical treatment of CTS. There were no significant differences in clinical results between 2 groups.

Keywords: Carpal tunnel syndrome; Open surgery; Carpal tunnel release; Local anesthesia; General anesthesia

\section{Introduction}

Carpal tunnel syndrome (CTS) is a common neuropathy, affecting the median nerve as it passes under the transverse carpal ligament [1]. CTS is described as a nerve compression at the wrist plane, CTS causes numbness and tingling in the hand and fingers. Sir James Paget defined CTS first in1853, and since then, in the 1950s a scientist named George Phalen popularized the diagnosis and treatment of CTS [2-4]. The aetiology of CTS is considered idiopathic in most cases but it is still controversial $[5,6]$.

Conservative treatment consists of splinting or corticosteroid injections and surgical release of the carpal tunnel are the treatment method options [7-9]. There has been continued debate over the optimal management of this disease. Decision of the surgeon has consistently varied $[10,11]$.

Carpal tunnel release (CTR) is known as an effective treatment for idiopathic CTS. CTR is performed with a variety of techniques such as endoscopic (ECTR) or open (OCTR). Literature has not got consensus on the superiority of any one technique to another [10-12].

Local anaesthesia (LA) and general anaesthesia (GA) are anaesthetic options on the surgical treatment of CTS. LA is safe, fast, and effective, but the injection could be painful $[13,14]$. In one recent series, about $10 \%$ of patients indicated that they would prefer another form of anaesthesia [15]. When applying the local anaesthetics under the skin patient could have pain and pain could make the patient uncomfortable. Also from the surgeon side discomfort of the patient could affect the surgical procedure and sometimes visualization of the surgical area could be difficult due to oedema caused by local anaesthetics. These problems in surgical procedure could affect the clinical results of the surgery. Sedation or GA could make the procedure more comfortable [16].

The aim of the present study was to compare the effects of the anaesthetic methods on the patient's clinical results. To our knowledge this is the first report that compares the clinical outcomes of the open CTR with GA or LA.

\section{Materials and Methods}

Computerized patient database was searched to identify all patients with CTS who underwent open CTR between January 2009 and January 2013 at Fatih Sultan Mehmet Training and Research Hospital. The year 2013 was selected to ensure a minimum one-year follow-up. At the result of the search total of 148 patients (169 CTS hands) were found.

Of the 148 patients (169 CTS hands) 21 patients were operated bilaterally and excluded from study. Of the 127 patient operated monolaterally. One patient died from another reason and 14 patients was not available and excluded from the study. Fifty five patients operated with GA (group A) and 57 patients with LA (group B). The last available 50 monolateral patient operated by the same surgeon for each group included to the study (Figure 1). 


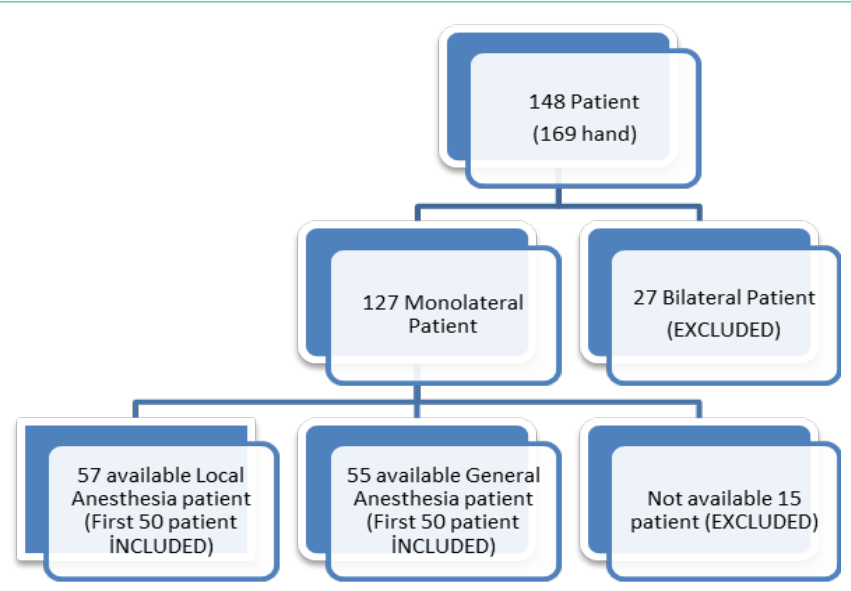

Figure 1: Figure showing patient inclusion criteria of the study.

Table 1: Table showing demographic data of patients included in this study.

\begin{tabular}{|c|c|c|}
\hline & $\begin{array}{c}\text { GROUP A GENERAL } \\
\text { ANAESTHESIA }\end{array}$ & $\begin{array}{c}\text { GROUP B LOCAL } \\
\text { ANAESTHESIA }\end{array}$ \\
\hline Number of participants & 50 & 50 \\
\hline MEN & 12 & 15 \\
\hline WOMEN & 38 & 35 \\
\hline Number of hands & 50 & 50 \\
\hline RIGHT & 37 & 36 \\
\hline LEFT & 13 & 14 \\
\hline $\begin{array}{c}\text { Hand involved: dominant/ } \\
\text { non dominant }\end{array}$ & $35 / 15$ & $38 / 12$ \\
\hline Diabetes & 6 & 5 \\
\hline Mean Age & $51.4(31-78)$ & $54.4(28-81)$ \\
\hline Mean Follow up (months) & 17.5 & 18.9 \\
\hline Smoking Status & 9 & $24 / 26$ \\
\hline $\begin{array}{c}\text { EMG results moderate/ } \\
\text { severe }\end{array}$ & $21 / 29$ & 9 \\
\hline
\end{tabular}

The CTS diagnosis was made by clinical examination and electromyography in all patients. Patients unresponsive to conservative treatment with wrist brace and anti-inflammatory medicine had CTR surgery. Patients with at least 12 months followup were included in the study. To assess the adequacy of treatment, we asked the patients to identify any difference between preoperative and postoperative symptoms on a visual analogue scale (VAS). Preoperatively 0 points indicated no pain and 10 were worst pain and postoperatively 0 points indicated absolute regression of symptoms and 10 points indicated lack of any effect of surgical treatment. Visual Analog Patient Satisfaction Scale (VAPSS) was used for comparison the level of patient satisfactory from surgical procedure. 10 point shows the highest satisfaction and 0 is the lowest or no satisfaction.

The surgeries were performed by the same surgeon using the same technique of OCTR. Before the surgery a standard antibiotic was used including $1 \mathrm{gr}$ cefazolin intravenously. LA with bupivacaine hydrochloride $(5 \mathrm{mg} / \mathrm{mL})$ and epinephrine $(5 \mu \mathrm{g} / \mathrm{mL})$ was used for anaesthesia in all cases in group B. The maximum dose never exceeded $5 \mathrm{~mL}$.

The length of the skin incision beginning from distal wrist crease and extending distally about 2 to $2.5 \mathrm{~cm}$ over the carpal canal was made. Bipolar diathermy was used for haemostasis. The flexor retinaculum was incised until the distal end with scissors and epineural neurolysis was performed when deemed appropriate. Skin was closed using single sutures. A neutral-position wrist splint was used for postoperative immobilization for two weeks. We attempted to contact patients by searching medical records and personsearching services.

\section{Statistical analysis}

Data analysis was performed using SPSS statistical software version 22.0 for Windows (SPSS Inc., Chicago, USA). The data are shown as mean \pm Standard deviation for continuous variables, median (minimum-maximum) for ordinal ones, and frequency with percent for categorical ones. Means were compared with using Student's $t$ test. Categorical comparisons were made using chisquare test, where appropriate. $P$ value less than 0.05 was considered statistically significant.

\section{Results}

The mean age of the patients was 51.4 (31-78) years in Group A and $54.4(28-81)$ years in Group B. There was no statistically significant difference between groups in terms of age $(p=0.362)$. There were 38 (76\%) females and 12 (24\%) males in Group A, and 35 $(70 \%)$ females and $15(30 \%)$ males in Group B ( $p=0.055)$.

There was difference between groups in terms of laterality $(\mathrm{p}=$ 0.01 ). The mean follow-up was not significantly different between two groups 17.5 (SD 1.4) months in Group A and 18.9 (SD 2.0) months in Group B ( $p=0.062)$. Intraoperative complications such as nerve or vessel injury were not observed in each group. In one diabetic patient superficial wound infection that healed with oral antibiotic administration was observed in group $B$.

Preoperatively, 47 (51\%) patients in Group A and 45 (90\%) patients in Group B complained of night pain. Postoperatively, there was not a significantly difference between groups in night pain. It was detected nine (18\%) hands in Group A eight (16\%) hands in Group $\mathrm{B}(\mathrm{p}=0.357)$. The night pain complaint was not significantly reduced after CTR in both groups ( $>0.21$ for both).

There was significantly different on the average time of surgery. It was 17.9 (SD 2.1) minute in group A and 14.8 (SD 1.7) minute in group B (P: 0.01). Also the average discharge time from hospital was significantly different. The time was 22.4 (SD 1.9) hours in group A and 3.6 (SD 0.9) in group B (P: 0.01). At final follow-up there were no recurrences or new surgical exploration. Average time to return to daily life activities and work were 10.8 (SD 1.5) and 11.2 (SD 1.3) days, there was no statistically significant difference between groups $\mathrm{A}$ and $\mathrm{B}$ in term of return to daily activity (P: 0.173 ).

Average time of surgery was significantly different between two groups. The average time of surgery was 17.9 (SD 2.1) minute in group A and 14.8 (SD 1.7) minute in group B (P: 0.01). At final follow-up there were no recurrences or new surgical exploration. Average time to return to daily life activities and work were 10.8 and 11.2 days, there was no statistically significant difference between groups $A$ and $\mathrm{B}(\mathrm{P}: 0.173)$.

A total of 6 patients (12\%) were diabetic in group A, whereas $5(10 \%)$ were diabetic in group B (p: 0.09). Retrospectively EMG 


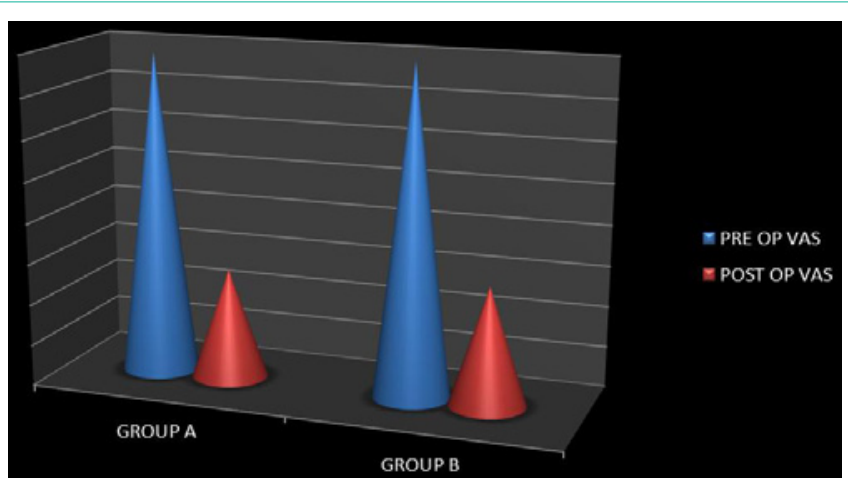

Figure 2: Figure showing preoperative and postoperative visual analogue score of group A and group B.

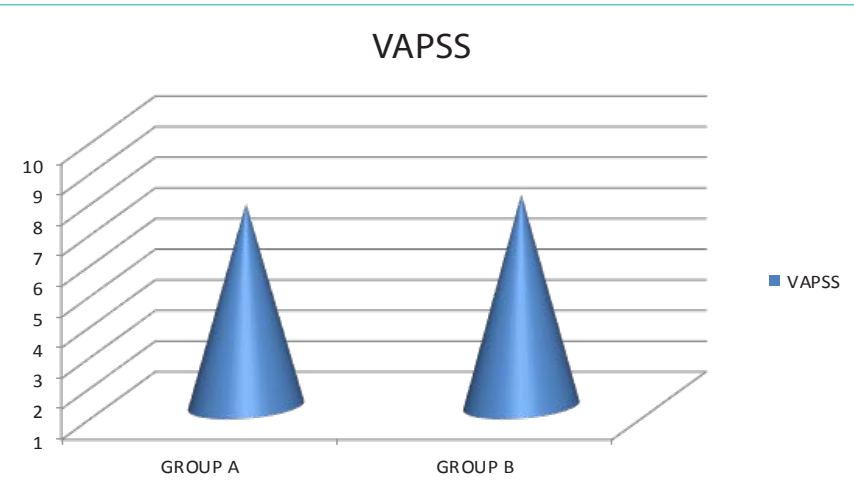

Figure 3: Figure showing visual analogue patients satisfaction score of group $A$ and group $B$.

results were similar between group A and B. In group A 21 patient was moderate and 29 patients was severe whereas 24 patients were moderate and 26 patients was severe (P: 0.500) (Table 1 ).

Preoperatively, the mean VAS score was 7,9 (SD 1.2) in Group A and significantly decreased to 2.7 (SD1.7) $(\mathrm{p}=0.01)$ also at the Group $B$ the mean VAS score significantly decreased 8,0 (SD 1.9) to 2.9 (SD 1.1) $(\mathrm{p}=0.01)$ (Figure 2). Between the groups the postoperative VAS score was not significantly different $(\mathrm{p}=0.112)$ also VAPSS score was not significantly different $(\mathrm{p}=0.158)$ (Figure 3 ).

\section{Discussion}

The most commonly performed surgical technique in the treatment of CTS is relieving the median nerve via cutting the transverse carpal ligament. Sufficient release of the carpal ligament is essential, and there could be possible anatomic variations. Postoperative complications, including excessive scar tissue, injury to the palmar motor branch of the median nerve, and unsatisfactory release, may result in an increase in the patient's complaints $[17,18]$. In the present study, we did not encounter such problems in either study group.

The learning curve of the surgical methods must be taken into consideration when evaluating results and outcome. CTS surgery requires proper training and experience before proficiency is reached $[19,20]$. In experienced or careless hands, the outcomes for all techniques may be similar and have low morbidity $[20,21]$. In our study there was no surgical learning curve and all surgical procedures made by experienced senior author (BS).

El Maraghy and Devereauux conducted a survey of orthopaedic and plastic surgeons in Ontario, Canada, to identify variations in the choice of surgical setting and anaesthesia when treating CTS [22]. Surveys were delivered to 606 orthopaedic and plastic surgeons; $75 \%$ responded to the questionnaire. The authors found that orthopaedic surgeons used the formal operating room for all CTR surgeries significantly more than plastic surgeons. Also in the selection of anaesthesia method there were significant differences between the two specialties. Orthopaedists used regional or GA more compared to plastic surgeons. In our Serie 68 operations were made with GA and 73 with LA but all in operating room.

In a published literature antibiotic using rate was found $46.7 \%$ around orthopaedic surgeons before the surgical procedure [23]. In our study we had a standard procedure for using antibiotics. Our low infection rate could be related with this.

GA could have more comorbidities rather than LA including respiratory problems [24]. In our study comorbidities due to GA was very low. This could be related with short surgical procedure time.

Discharge time from the surgery is important for the patients. Faster discharge from the hospital will decrease the hospital costs and will increase the patient satisfaction. In our study GA group has a significant longer discharge time after surgery. In addition, cost is lowered further because preoperative screening tests (blood tests, chest X-rays and electrocardiographs) are not required within LA [25]. Also local regulations do not require an anaesthesia nurse or doctor for its use, economically LA is probably more cost effective than GA. Despite these factors, there is a role for GA in extremely anxious patients, or when additional supple-mental procedures are anticipated or planned. This study shows that both methods of anaesthesia are well tolerated and are of value to the hand surgeon.

An important limitation of this research was to be a retrospective study. The retrospective character of the study implies that in some patients the operation was done several years before the questionnaire was completed, which could potentially affect the outcome. However, as the period of time between CTR and answering of the questionnaire was relatively similar between the two groups of patients. Also that study had potential limitation: reported complications were in-hospital. Another important limitation is: it was not a randomized controlled trial, despite statistical corrections; unaccounted (unobserved) factors could have biased treatment exposure (to regional or general anaesthesia).

\section{Conclusion}

All patients had a significant resolution of CTS symptoms at the final follow up, as assessed by the VAS and VAPSS. The main objective of this study was to compare the results of CTR under GA and LA. A high percentage of patients reported high levels of satisfaction, excellent results and improvements in their quality of life in both group. As the VAS and VAPSS were considered the primary outcome measure, the results of our study show that the type of anaesthesia has no effect on the results of surgical treatment of CTS. Further prospective, controlled, high-powered, and randomized 
studies comparing these two treatment options need to be performed to determine the differences in patient outcomes.

\section{References}

1. Phalen GS. The carpal-tunnel syndrome. Seventeen years' experience in diagnosis and treatment of six hundred fifty-four hands. J Bone Joint Surg Am. 1966; 48: 211-228.

2. Paget J. Lectures on surgical pathology. Philadelphia: Lindsay \& Blakiston; 1854.

3. Phalen GS, Gardner WJ, La Londe AA. Neuropathy of the median nerve due to compression beneath the transverse carpal ligament. J Bone Joint Surg 1950; 32: 109-112.

4. Phalen GS, Kendrick JI. Compression neuropathy of the median nerve in the carpal tunnel. J Am Med Assoc. 1957; 164: 524-530.

5. Geoghegan J, Clark D, Bainbridge L, Smith C, Hubbard R. Risk factors in carpal tunnel syndrome. Journal of Hand Surgery. 2004; 29: 315-320.

6. J. E. Farmer and T. R. Davis. Carpal Tunnel Syndrome: A Case-Contro Study Evaluating Its Relationship with Body Mass Index and Hand and Wrist Measurements. J Hand Surg Eur Vol, 2008; 4: 445-448.

7. Weiss APC, Sachar K, Gendreau M. Conservative management of carpa tunnel syndrome: a re-examination of steroid injection and splinting. The Journal of Hand Surgery, 1994; 19: 410-415

8. Racasan $\mathrm{O}$, Dubert $\mathrm{T}$. The safest location for steroid injection in the treatment of carpal tunnel syndrome. J Hand Surg Br. 2005; 30: 412-414.

9. Huisstede BM, Randsdorp MS, Coert JH, Glerum S, van Middelkoop M, Koes BW. Carpal tunnel syndrome. Part II: effectiveness of surgical treatments - a systematic review. Arch Phys Med Rehabil. 2010; 91: 1005-1024.

10. Atroshi I, Hofer M, Larsson GU, Ornstein E, Johnsson R, Ranstam J. Open compared with 2-portal endoscopic carpal tunnel release: a 5-year follow-up of a randomized controlled trial. J Hand Surg Am. 2009; 34: 266-272.

11. Brown RA, Gelberman RH, Seiler JG 3rd, Abrahamsson SO, Weiland AJ, Urbaniak JR, et al. Carpal tunnel release. A prospective, randomized assessment of open and endoscopic methods. J Bone Joint Surg Am. 1993 75: $1265-1275$.

12. Buch-Jaeger N, Foucher G. Correlation of clinical signs with nerve conduction tests in the diagnosis of carpal tunnel syndrome. J Hand Surg Br. 1994; 19: $720-724$.
13. Katz RD, La Porte DM. Use of short-acting local anesthetics in hand surgery patients. J Hand Surg [Am]. 2009; 34: 1902-1905.

14. Koay J, Orengo I. Application of local anesthetics in dermatologic surgery. Dermatol Surg. 2002; 28: 143-148.

15. Koegst WH, Wolfle O, Thoele K, Sauerbier M. The "Wide Awake Approach" in hand surgery: a comfortable anaesthesia method without a tourniquet. Handchir Mikrochir Plast Chir. 2011; 43: 175-180.

16. Pollock H, Forman S, Pollock T, Raccasi M. Conscious sedation/loca anesthesia in the office-based surgical and procedural facility. Clin Plast Surg. 2013; 40: 383-388.

17. Benson LS, Bare AA, Nagle DJ, Harder VS, Williams CS, Visotsky JL. Complications of endoscopic and open carpal tunnel release. Arthroscopy. 2006; 22: 919-924.

18. Palmer AK1, Toivonen DA. Complications of endoscopic and open carpa tunnel release. J Hand Surg Am. 1999; 24: 561-565.

19. Litin SC, Prickman JL. Carpal tunnel syndrome. Mayo Clin Updat. 1993; 9 $1-2$.

20. Matthias Vanhees, Francis Van Glabbeek, Peter C. Amadio. Carpal Tunne Syndrome etiology update: where do we stand? Acta Orthop Belg. 2014; 80 331-335

21. Rab M, Grunbeck M, Beck H, Haslik W, Schrögendorfer KF, Schiefer HP et al. Intra-individual comparison between open and 2-portal endoscopic release in clinically matched bilateral carpal syndrome. J Plast Reconstr Aesthet Surg. 2006; 59: 730-736.

22. EIMaraghy A, Devereaux MW. Variability in the surgical management of carpal tunnel syndrome: implications for the effective use of healthcare resources. Healthc Q. 2009; 12: 85-91.

23. Shin EK, Bachoura A, Jacoby SM, Chen NC, Osterman AL. Treatment of carpal tunnel syndrome by members of the American Association for Hand Surgery. Hand (N Y). 2012; 7: 351-356.

24. Kawashima Y1, Takahashi S, Suzuki M, Morita K, Irita K, Iwao Y. et al. Anesthesia-related mortality and morbidity over a 5-year period in 2,363,038 patients in Japan. Acta Anaesthesiol Scand. 2003; 47: 809-817.

25. Derkash Rs, Weaver JK, Berkeley ME, Dawson D. Open carpal tunnel release with wrist block and wrist tourniquet. Orthopaedics 1996; 19: 589-590.
Austin J Musculoskelet Disord - Volume 4 Issue 2 - 2017

ISSN : 2381-8948 | www.austinpublishinggroup.com

Karaca et al. ( All rights are reserved
Citation: Karaca S, Akpolat AO, Oztermeli A, Bildik C, Erdem MN and Saygi B. Comparison of Local versus General Anaesthesia for Carpal Tunnel Release. Austin J Musculoskelet Disord. 2017; 4(2): 1043. 